was found that a liquid has to be regarded as a broken up crystal in which there is thermodynamic equilibrium between heat motion and forces of crystal formation. ${ }^{2}$ It was seen both theoretically and from observations on X-ray diffraction that there is a maximum in the molecular arrangements in liquids corresponding to the structure of the substance in the crystal state. It is evident that this partial crystallinity of liquids is responsible for the modified scattering found by Gross. It is, therefore, expected that those liquids which produce sharp diffraction effects should also produce sharp modified lines, and it will be interesting to see whether that comes out true.

In the same paper Gross suggests an instructive explanation of the broadening of Raman lines: that each Raman line consists of a number of unresolved lines modified further by the elastic heat waves; he supports this view also in his later paper. ${ }^{3}$ This does not appear to me sufficient to explain the fact that in some cases where he observed modified scattering due to elastic waves there is no broadening of the Raman lines. The following explanation appears probable to me. In a crystal the natural frequencies of a molecule that are responsible for the Raman effect are modified, due to the surrounding molecules; but on account of the regular arrangement all the molecules are affected in the same manner, and hence Raman lines are sharp. But in a liquid the regularity of arrangement is broken, and hence the broadening out of the natural frequencies of the molecules. We may, therefore, say generally that the more diffuse the X-ray diffraction of a liquid the broader are its Raman lines, provided the natural frequencies of molecules are sufficiently influenced by the surrounding molecules.

\section{Kedareswar BanerJee.}

Alipore Observatory, Calcutta, Nov. 15.

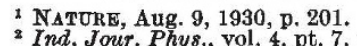

${ }^{2}$ Ind. Sour. Phys., vol. 4, pt. 7, pp. 541-556.

- NATURE, Oct. 18,1930 , p. 603 .

\section{The Photo-Reaction of Hydrogen and Iodine Monochloride.}

WE are unable to accept the conclusions of Rollefson and Lindquist 1 that hydrogen and iodine monochloride do not react at ordinary temperatures under the influence of light. These authors maintain that the reaction $\mathrm{H}_{2}+\mathrm{Cl}=\mathrm{HCl}+\mathrm{H}$ does not readily take place because the $\mathrm{Cl}$ atoms resulting from the photodissociation of $\mathrm{ICl}$ are mostly in the unexcited state. They consider that the accompanying reaction $\mathrm{ICl}+\mathrm{Cl}=\mathrm{I}+\mathrm{Cl}_{2}$ is more probable. We had already concluded certain experiments with $\mathrm{H}_{2}$ and $\mathrm{ICl}$ before the above paper appeared, and had found, as we now find on repeating the work, that $\mathrm{H}_{2}$ and $\mathrm{ICl}$ reacted rapidly in strong light if the hydrogen pressure were large compared with the pressure of ICl. This must mean that conditions favour the greater probability of the reaction $\mathrm{H}_{2}+\mathrm{Cl}=\mathrm{HCl}+\mathrm{H}$, and that excited $\mathrm{Cl}$ atoms may not be necessary for it to take place. The idea, however, that ICl dissociates into normal atoms is not accepted now by the original investigators. ${ }^{2}$

In some of our preliminary experiments we passed carefully purified hydrogen at atmospheric pressure and ICl at a pressure of about $20 \mathrm{~mm}$. into thin glass bulbs, which were then sealed off. The reaction was very slow in artificial light (electric globe), more rapid in diffuse daylight, and very rapid in direet sunlight, $\mathrm{I}_{2}$ and $\mathrm{HCl}$ being the main products. We have also used hydrogen filtered through palladium and dried over phosphoric oxide, and Kahlbaum's purest, crystalline ICl. No special precautions were taken to eliminate possible inhibitors except oxygen. It is easy to understand why the reaction proceeds very slowly compared with the hydrogen-chlorine reaction, as the chain will terminate with the formation of iodine atoms.

We hope to publish some quantitative results very shortly.

D. P. Melicor.

University of Sydney,

N.S.W., Australia.

1 Jour. Amer. Chem. Soc., 52, $2793 ; 1930$.

- Gibson, NATURE, 123, 347; 1929.' Zeit. für Physik, 50, 692 ; 1928.

\section{Crystal Structure of Molybdenum Trioxide.}

Crystals of molybdenum trioxide, prepared by subliming $\mathrm{MoO}_{3}$ powder, obtained from the pure ammonium compound, have been examined with $\mathrm{X}$-rays. The crystals were small, thin, lustrous plates, parallel to $b(010)$.

Laue photographs showed orthorhombic symmetry. Oscillation photographs about the three principal axes gave the cell size as

$$
\begin{aligned}
& a=3.93 \pm 0.02 \mathrm{~A} . \\
& b=13.91 \pm 0.05 \mathrm{~A} . \\
& c=3.57 \pm 0.02 \mathrm{~A} .
\end{aligned}
$$

These seem to bear no relation to the crystallographic axial ratio given by Nordenskiold,

$$
a: b: c=0.3874: 1: 0 \cdot 4747 \text {. }
$$

There are four molecules of $\mathrm{MoO}_{3}$ per cell. The space group is $Q_{h}^{16},(\mathrm{bnm})$, since $(h 01)$ is halved if $h+1$ is odd, and $(0 k 1)$ is halved if $k$ is odd. The coordinates of the molybdenum atoms are as follows, the centre of symmetry being taken as origin : $u v \frac{1}{4}: \bar{u}+\frac{1}{2}, v+\frac{1}{2}, \frac{1}{4} ; u+\frac{1}{2}, \bar{v}+\frac{1}{2}, \overline{\frac{1}{4}} ; \bar{u} \bar{v} \frac{\overline{1}}{4}$. $u=36^{\circ}, v=30^{\circ}$, approximately.

Intensity measurements are being made on an ionisation spectrometer, to fix the oxygen positions.

A full account of the structure will appear later in the Zeitschrift für Kristallographie.

The Mineralogical Laboratory, NORA WOOSTER.

The Museums, Cambridge,

$$
\text { Dec. } 8 .
$$

\section{The Emission Bands of Sulphur.}

H. H. VAN IDDEKINGE ${ }^{1}$ has made the interesting observation that in the emission spectrum of sulphur produced by him, such of the bands as were found by Rosen $^{2}$ and Henri and Teves ${ }^{3}$ to be diffuse in the absorption spectrum, are entirely absent. This gives strong evidence in favour of the view of "predissociation' in the molecule.

A few months ago, when I was working at King's College, London, I obtained spectrograms of the emission bands of sulphur by exciting them in a discharge tube containing sulphur vapour in the presence of high pressure argon. Analysis of the bands is in progress, but $\mathrm{I}$ find on my plates all the bands recorded in absorption by previous workers and many more towards the red end, and the bands are all sharp. This indicates that argon helps in some way to arrest the pre-dissociation of the sulphur molecule. Analogous influences of rare gases are known, for example, in the production of the Cameron bands, the Baldet-Johnson bands, etc.

In addition to the already known bands attributed to sulphur in the visible and the ultra-violet, a few new bands degraded towards the shorter wavelength side have been also photographed in the extreme ultra-violet beginning from about $\lambda 2100$ 